\title{
Left Bundle Branch Block in a 29-Year-Old Non-Competitive Runner
}

\author{
Shayef Gabasha, ${ }^{a}$, Theresa Ratajczak ${ }^{\mathrm{a}}$
}

\begin{abstract}
Left bundle branch block (LBBB) is an electrocardiogram (EKG) abnormality that represents disruption of the normal electrical conduction system. While commonly found in patients with cardiomyopathies, it may manifest even in the absence of any other cardiac disease. Transient LBBB is likely a common intermediate step in the progression from a normal EKG to chronic LBBB. Given that a chronic LBBB portends poor outcomes, transient LBBB may be an early indication to initiate cardiopulmonary evaluation. We present a case of a 29-year-old Caucasian male with multiple admissions for chest pain which progressed from a normal EKG to transient LBBB, to chronic LBBB over a 2-year period.
\end{abstract}

Keywords: LBBB; Athlete; Physiologic remodeling; RBBB; Cardiomyopathy

\section{Introduction}

Left bundle branch block (LBBB) is a common electrocardiographic (EKG) abnormality that is defined as QRS duration greater than $120 \mathrm{~ms}$, notched $\mathrm{R}$ wave in leads I, V5 and V6, rS or QS in V1 and absence of Q waves in the left lateral leads [1]. In healthy males, the risk of developing LBBB during their lifetime is $0.7 \%$ [2]. In patients that develop LBBB, $54 \%$ have no previous EKG abnormalities, while $3.5 \%$ have an antecedent intermittent or transient LBBB [2]. This EKG pattern is commonly found in patients with known cardiomyopathy. However, young athletes do not commonly present with this abnormality [3]. In a screening study of 32,561 high school athletes, 817 athletes had EKG abnormalities, none of which included LBBB [4]. Structural and electrical remodeling of the athlete's heart in response to the physiological demand of exercise is a wellrecognized phenomenon which leads to subsequent benign

Manuscript accepted for publication February 06, 2017

${ }^{a}$ Wright State University, 128 East Apple St., 2nd Floor, Dayton, OH 45409, USA

${ }^{\mathrm{b} C}$ Corresponding Author: Shayef Gabasha, Wright State University, 128 East Apple St., 2nd Floor, Dayton, OH 45409, USA. Email: shayef120@gmail.com

doi: https://doi.org/10.14740/jmc2760w and reversible EKG changes [5]. These EKG changes include left ventricular hypertrophy (LVH), right bundle branch block (RBBB), sinus bradycardia, first degree atrioventricular block, and domed ST elevations. LBBB however, is not recognized as a normal EKG variant in the athlete heart. In fact, in a 29 -year follow-up study of 3,983 healthy young males, development of LBBB was associated with a tenfold increase in sudden cardiac death. Additionally, some studies have reported a mean survival of less than 5 years after documentation of LBBB in patients with known cardiomyopathies. It comes as no surprise that current guidelines consider LBBB an EKG abnormality that warrants further evaluation which should include cardiac imaging [2]. Kim et al emphasize in their review of the literature that LBBB is not likely associated with the physiological stress related changes secondary to exercise [5]. We present a case of a 27-year-old male non-competitive runner whose EKG progressed from intermittent LBBB to persistent LBBB in a 2-year period without any underlying cardiac abnormalities.

\section{Case Report}

A previously healthy 27 -year-old male presented to the emergency room (ER) with chest pain. He was found to have normal vitals and clinical exam.

Basic labs and initial EKG (Fig. 1a) were unremarkable. Serial cardiac enzymes were also unremarkable. Telemetry and serial EKG (Fig. 1b) monitoring revealed an intermittent wide complex rhythm without tachycardia consistent with a transient LBBB. Evaluation with stress echo was negative for valve disease, wall motion abnormalities or functional abnormality. He was treated for presumed anxiety and discharged home. An EKG prior to discharge (Fig. 1c) showed resolution of the transient LBBB. Two years later, the patient presented to the ER with atypical chest pain. His vitals and clinical exam were within normal limits. Basic labs, serial cardiac enzymes, drug screen and CXR were unremarkable. Lipid panel showed LDL of 125.

EKG (Fig. 2a) and telemetry revealed a persistent LBBB with a normal heart rate at presentation in the ER. The patient's chest pain resolved with administration of antacids. He was evaluated by cardiology in the ER and coronary angiography was not pursued due to resolution of symptoms with antacids. Furthermore, the patient was noted to be an avid runner who runs 20 - 30 miles per week. He was admitted for serial cardiac enzymes and observation of symptoms and was placed on te- 


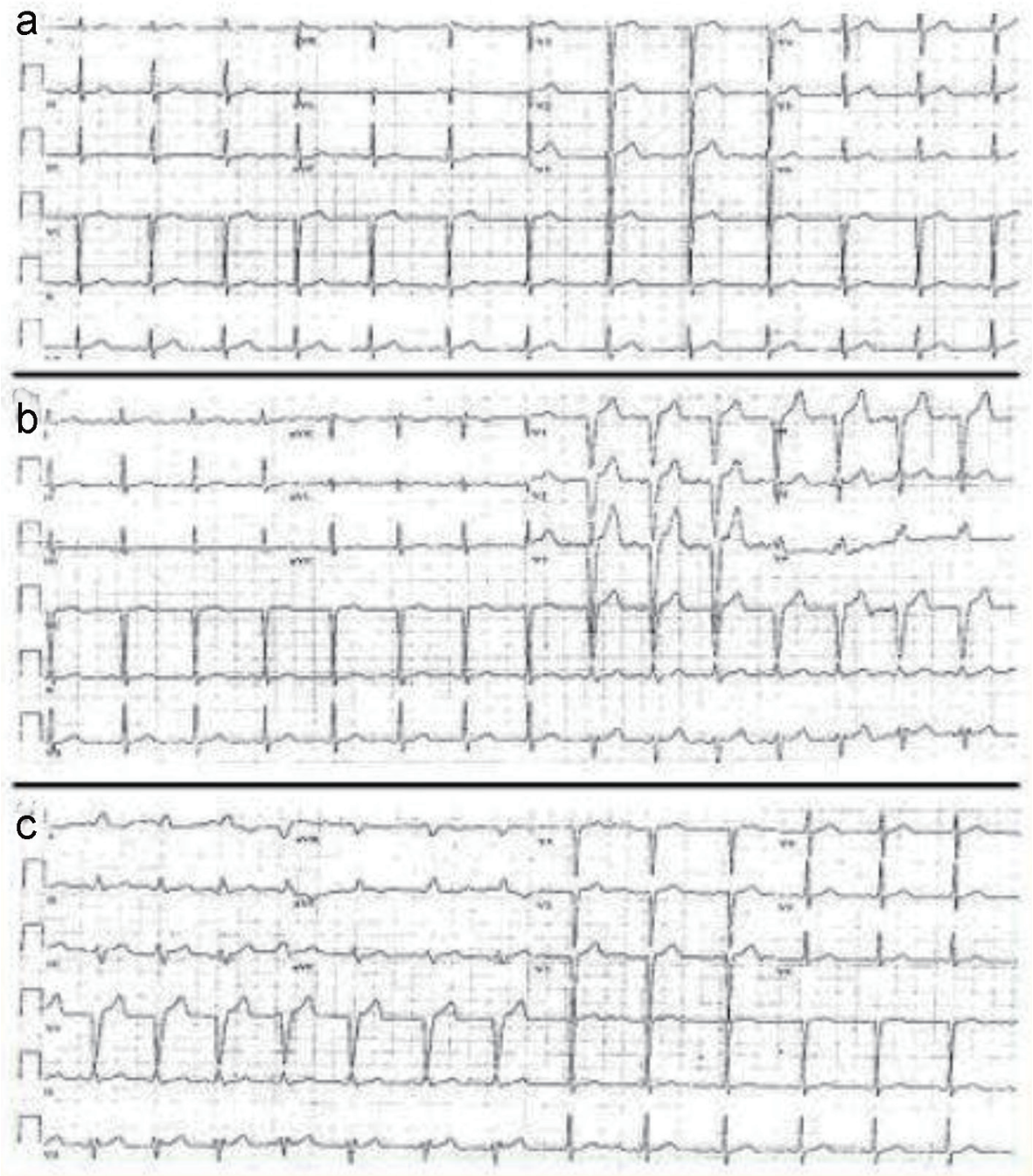

Figure 1. (a) Admission EKG shows normal sinus rhythm. (b) EKG captures transition to LBBB. (c) EKG captures transition from LBBB to normal sinus rhythm.

lemetry. Serial cardiac enzymes remained within normal limits and serial EKGs remained stable. Telemetry during the $48 \mathrm{~h}$ observation period remained consistent with LBBB without any arrhythmias.

Ischemic evaluation with $2 \mathrm{D}$ echo (Fig. 2b, c) and chemical MPI (Fig. 3) showed ejection fraction (EF) of 50-55\% with no reversible or fixed defects. He was diagnosed with gastroesophageal reflux disease (GERD) in view of his clinical presentation and symptom resolution with antacids. The patient was discharged home in good condition and without any limitations.

\section{Discussion}

While ischemic and non-ischemic cardiomyopathies are the most concerning diagnoses associated with $\mathrm{LBBB}$, there have been many case reports of LBBB in the absence of cardiac disease $[7,8]$. Elterman et al summarize at least 11 cases of non-ischemic LBBB in their brief discussion of a perioperative LBBB case report [7]. These cases included left heart catheterization-induced transient LBBB, rate-dependent LBBB, physiologic maneuver-induced LBBB as well as perioperative transient. However, upon brief review of the literature using the mesh terms "athlete" and "LBBB" using Pubmed central, there were no case reports nor discussions of LBBB as a benign finding in young healthy athletes. It is likely that the association is exceedingly rare, but remains a possibility.

In order to understand the disease processes associated with LBBB, it is essential to highlight the mechanism behind the abnormal EKG morphology. Essentially, LBBB morphology appears on an EKG tracing due to a prolonged myocardial activation process. The electrical conduction can be either anterograde through the HIS-Purkinje system, yet delayed, or reaches the His-Purkinje system in a retrograde fashion [7]. As discussed above, this can be disrupted by a physiologic or a structural abnormality at the right location. Given the established physiological stress remodeling paradigm discussed above, it is plausible 


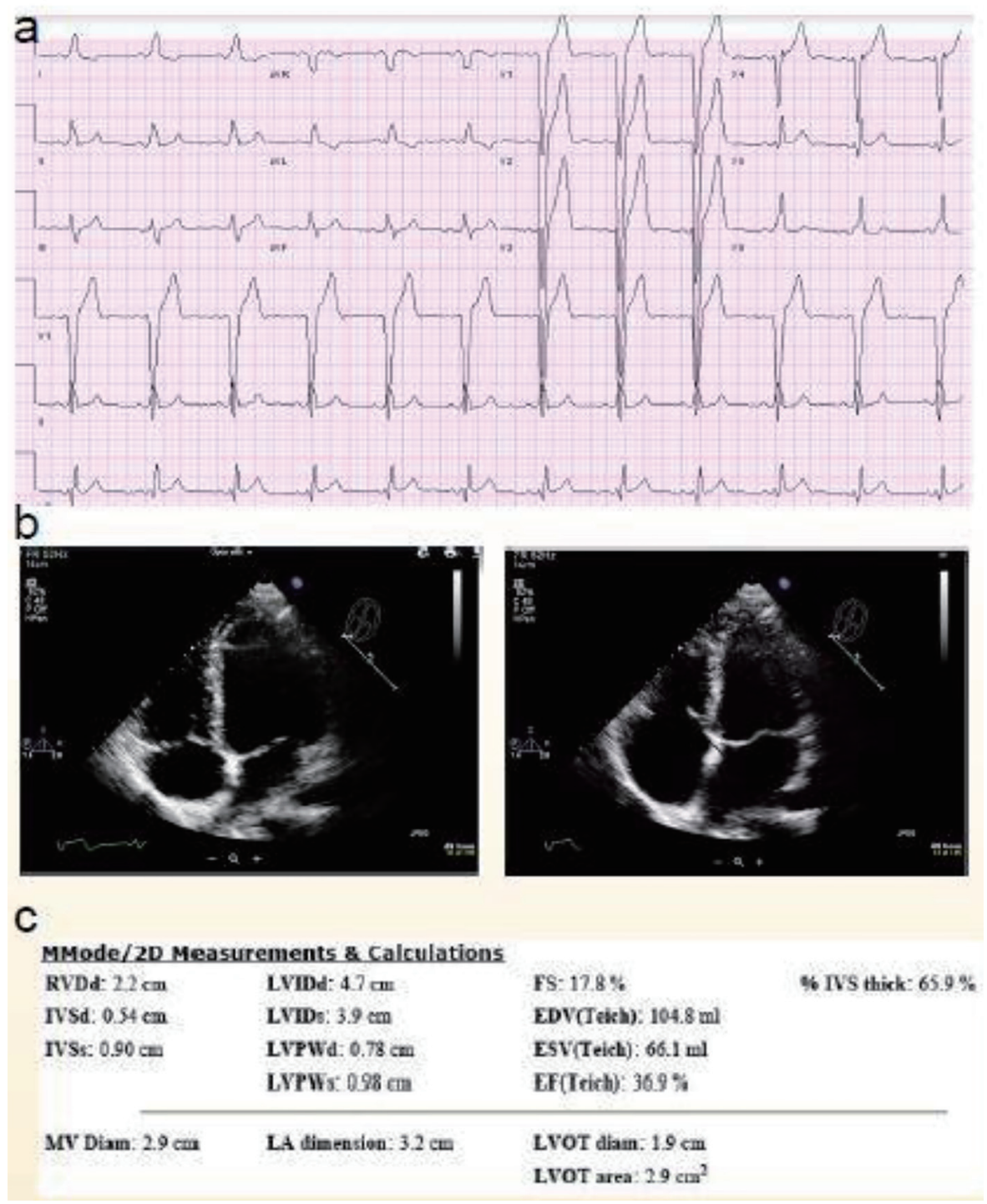

Figure 2. (a) Second admission EKG with LBBB. (b) 2D echo images in systole and diastole. (c) $2 \mathrm{D}$ echo measurements and calculations.

that there is a connection, although likely exceedingly rare, between an LBBB and normal physiological remodeling. For example, one study showed that $27.4 \%$ of professional basketball athletes met LVH criteria by echocardiography [9]. This study, which included 529 athletes, does not mention any EKG associations. However, given that LVH is associated with RBBB in athletes, the mechanism that causes RBBB may be able to cause LBBB. Additionally, this remodeling process, which is thought to be secondary to the stress of exercise, is thought to be reversible via a "detraining" period where athletes cease all exercise training activities [5]. The rarity of the theoretical association between LBBB and LVH is highlighted by the fact that in over 32,000 young athletes, no LBBB abnormality was found. It comes as no surprise that multiple authors, including Kim et al, state that LBBB is not likely associated with normal stress exercise physiological changes [6].

The limitation of this case is that the patient did not com- plete left heart catheterization or cardiac MRI. However, a stress echocardiogram, a resting echocardiogram, as well as a stress myocardial perfusion imaging (MPI) all conclusively ruled out a cardiomyopathy. While we recognize that a rare abnormality could theoretically have been missed by the testing modalities obtained, it is very unlikely in view of the patient's exceptional health and ability to complete extensive cardiovascular exercise without any symptoms. In view of the lack of family history of sudden cardiac death, lack of electrolyte abnormalities, and extensive cardiopulmonary workup, we concluded that this could be a very rare case of exerciserelated LBBB in an otherwise healthy non-competitive athlete. While we cannot make any conclusive statements from this case, being able to capture the transition from a normal EKG to intermittent $\mathrm{LBBB}$ to permanent $\mathrm{LBBB}$, over a 2-year period, offers us a unique vantage point, namely because the patient continued to exercise during this period without any other 


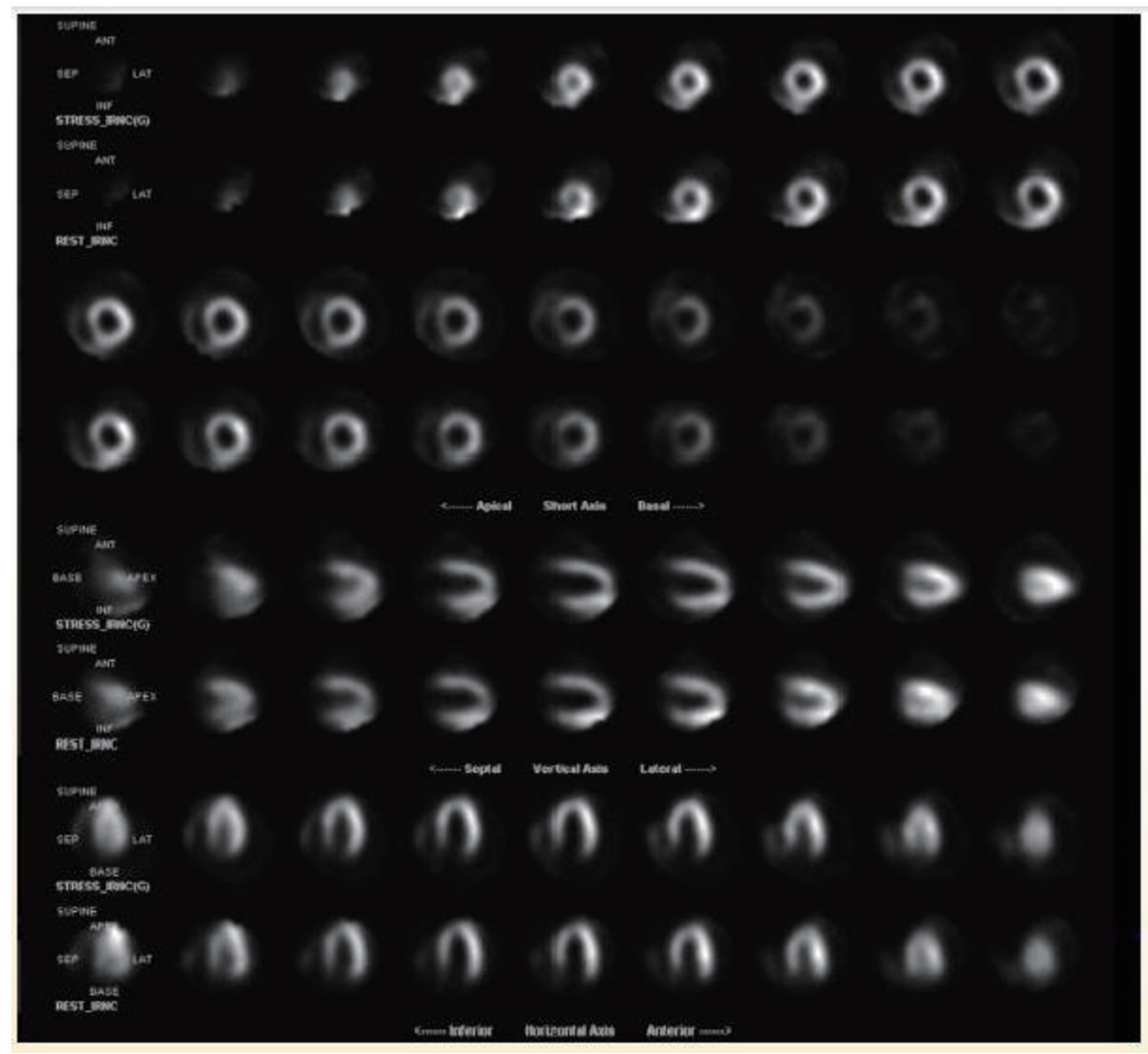

Figure 3. Lexiscan myocardial perfusion imaging without any reversible lesions concerning for ischemia.

significant illnesses or cardiac events. The prognostic implications of the presence of LBBB in a healthy young athlete have yet to be conclusively determined and further research is needed. Further research is also indicated to challenge the currently accepted paradigm that LBBB cannot be associated with the physiological stress response to exercise.

\section{References}

1. Neiger JS, Trohman RG. Differential diagnosis of tachycardia with a typical left bundle branch block morphology. World J Cardiol. 2011;3(5):127-134.

2. Rabkin SW, Mathewson FA, Tate RB. Natural history of left bundle-branch block. Br Heart J. 1980;43(2):164169.

3. Neeland IJ, Kontos MC, de Lemos JA. Evolving considerations in the management of patients with left bundle branch block and suspected myocardial infarction. J Am Coll Cardiol. 2012;60(2):96-105.
4. Marek J, Bufalino V, Davis J, Marek K, Gami A, Stephan W, Zimmerman F. Feasibility and findings of large-scale electrocardiographic screening in young adults: data from 32,561 subjects. Heart Rhythm. 2011;8(10):1555-1559.

5. Drezner JA, Fischbach P, Foelicher V, et al. Normal electrocardiographic findings: recognizing physiologic adaptations in athletes. Br J Sports Med. 2013;47.

6. Kim JH, Baggish AL. Electrocardiographic right and left bundle branch block patterns in athletes: prevalence, pathology, and clinical significance. J Electrocardiol. 2015;48(3):380-384.

7. Elterman KG, Mallampati SR, Tedrow UB, Urman RD. Postoperative episodic left bundle branch block. A A Case Rep. 2014;2(4):44-47.

8. Edelman JD, Hurlbert BJ. Intermittent left bundle branch block during anesthesia. Anesth Analg. 1980;59(8):628630.

9. Engel DJ, Schwartz A, Homma S. Athletic Cardiac Remodeling in US Professional Basketball Players. JAMA Cardiol. 2016;1(1):80-87. 